Volume 9, No.5, September - October 2020

International Journal of Advanced Trends in Computer Science and Engineering

Available Online at http://www.warse.org/IJATCSE/static/pdf/file/ijatcse128952020.pdf

https://doi.org/10.30534/ijatcse/2020/128952020

\title{
Segmentation and Classification of Brain Tumor from Magnetic Resonance Images Using K-Means Algorithm and Hybrid PSO-WCA Based Radial Basis Function Neural Network
}

\author{
T.Gopi Krishna ${ }^{1}$, Satyasis Mishra $^{2}$,Sunita Satapathy ${ }^{3}$, K.V.N. Sunitha ${ }^{4}$ \\ ${ }^{1}$ Dept. of CSE,SoEEC, Adama Science and Technology University,Ethiopia, gktiruveedula@ gmail.com \\ ${ }^{2}$ Dept. of ECE,SoEEC, Adama Science and Technology University,Ethiopia, , satyasis.mishra@ astu.edu.et \\ ${ }^{3}$ Dept.of Zoology, School of Applied Science, Centurion University of Technology \& Management, Odisha, \\ India, sunita.mishra@cutm.ac.in \\ ${ }^{4}$ Dept. of CSE, BVRIT Women's Engineering College, Hyderabad, India, k.v.n.sunitha@ gmail.com
}

\begin{abstract}
The manual detection and classification of the tumor becomes a rigorous and hectic task for the radiologists from magnetic resonance images. This paper presents a novel Hybrid PSO-WCA (Particle Swarm Optimization-Water Cycle Algorithm) based Radial Basis Function Neural Network (RBFNN) machine learning classification model for brain tumors classification. The K- means algorithm has been employed for segmentation and GLCM (Gray Level Co-occurrence Matrix) technique for feature extraction. The extracted features are aligned as input to the PSO-WCA based radial basis function neural network for the classification of brain tumors. The weights of the RBFNN are updated by the PSO-WCA (Particle Swarm optimization) algorithm and the centers of the RBFNN are chosen by K-means algorithm. Further, the malignant and benign tumors has been classified by Fast fuzzy c-means, KNN (Nearest neighborhood) algorithm, Fuzzy c means algorithm and K-Means algorithm by taking features as input for visual localization and the performance of the clustering classification has been presented. This research work considered the brain tumor MRI (Magnetic Resonance Image) Dataset-255 from Harvard medical school. The result obtained from the proposed hybrid PSO-WCA-RBFNN classification model shows better classification accuracy of $99.62 \%$ and comparison results with the PSO-RBFNN, WCA-RBFNN and LMS-RBFNN models are also presented.
\end{abstract}

Key words : Fuzzy c means algorithm, Fast fuzzy c means, K-Nearest neighbour, Particle Swarm optimization, Radial Basis Function Neural Network

\section{INTRODUCTION}

The brain tumors are of mainly two types as malignant and Benign. The brain tumors symptoms caused by brain tumors are such as hypertensions, headaches, vomiting, Vision problems, peripheral vision problems leads to eye ball reverse, paralysing of mouth leads to abnormal talk, gradual loss of sensation leads to improper walk, hearing problems etc. are all the symptoms are found one by one when the brain tumor starts growing. There are mainly two categories of brain tumors are there as per the research in medical study. Craniopharyngiomas [1] are the rare, noncancerous tumors which grows slowly and affects pituitary gland and other structures near the brain. As per the medical practitioner, If it is not operated in advance, the chances of survival becomes difficult for a tumor affected patient Noramalina Bt Abdullahet al. [2] in 2011, presented the classification results of brain tumor of $65 \%$ using wavelet (Daubechies (db4)) and Support Vector Machine (SVM). Mohd Fauzi Othman et al.2011, [3], uses Principal Component Analysis and Probabilistic Neural Network (PNN) and reported precision of 73 to $100 \%$ with varying spread values from 1 to 3 . Damodharan and Raghavan [4] have presented a precision of $83 \%$ utilizing neural network predicated classifier for encephalon tumor detection and relegation. Alfonse and Salem [5] have proposed SVM predicated classifier and expeditious Fourier transform (FFT) for automatic relegation of encephalon tumor from MR images. Kumar and Vijayakumar [6] reported a relegation precision of $94 \%$ utilizing principal component analysis (PCA) and SVM and claims classification accuracy of $94 \%$.Cui et al. [7] proposed a localized FCM with cerebrospinal fluid as input with spatial information and claimed precision between $83 \%$ to $95 \%$ and claim $83 \%$ to $95 \%$ accuracy. Sharma et al. [8] reported a highly efficient method based on "texture-primitive" features along with artificial neural network(ANN) and claims classification accuracy of $100 \%$. Zanaty [9] presented a hybrid type of approach with FCM for encephalon tumor segmentation and obtained precision of $90 \%$ at the noise level. 
Wang et al. [10] utilized a detection technique with the intensity in homogeneities in segmentation. Torheim et al. [11], claimed better presages and ameliorated clinical factors in comparison with "first-order statistical features" utilizing wavelet transform, and SVM's algorithm. Deepa and Arunadevi [12] utilized extreme learning machine (ELM) for classification and obtained an accuracy of $93.2 \%$. Chaddad [13] has used Gaussian mixture model (GMM) feature extraction method and obtained an accuracy of $97.05 \%$. Nilesh Bhaskarrao Bahadure et al [14] utilized support vector machine for classification and achieved $96.51 \%$ accuracy. The classification and detection of the brain tumors are presented by the researchers through dissimilar classifiers such as SVM[25,26],PNN, MLP[28] etc. and found classification results in terms of "accuracy" and "computational time" for the cancerous and noncancerous brain tumors. The literature survey shows different classification techniques, segmentation process for brain tumor detection but the clustering classification is yet not considered. In this present work we have presented the novel clustering classification of benign and malignant tumors. The segmentation process accomplished by the K-Means algorithm, Fuzzy c means and fast fuzzy c means algorithm and features are extracted through a popular "Gray Level Cooccurrence Matrix (GLCM)"[15] technique. The extracted features are applied to the, KNN [16],FCM and recently published Fast fuzzy c-means algorithm clustering algorithms for visual localization of classification. Further, a PSO-WCA based RBFNN classification model has been proposed for classification and corresponding results along with error calculations are presented. In this work, the weights of the RBFNN are updated by the PSO-WCA algorithm and the centres of the radial basis function are chosen by the $\mathrm{k}$ - means algorithm. It is found that the PSO-WCA-RBFNN outperforms well in comparisons to the existed classifiers.

This paper organizes as follows: the section- 2 presents the materials and methods which includes proposed model with PSO-WCA updation and Fast fuzzy c means algorithm, section 3 presents results of segmentation and classification, section 4 presents the discussion of the research and Section-5 presents conclusion followed by reference.

\section{MATERIALS AND METHODS}

\subsection{Research Flow Diagram}

The classification of brain tumor focusses through clustering algorithms. The work flow accomplished through the three steps. At the first step the images are segmented by the K-Means algorithm and GLCM technique has been utilized for feature extraction. During the second step proposed PSO-WCA based RBFNN model accepts the features as input for classification. In the third step, features are served as input to the existed KNN[17], Fast FCM,
K-Means clustering algorithm. The research folw diagram for the research is presented in Fig.1.

\subsection{Dataset}

The datasets-255 is considered from the public database (http://adni.loni.usc.edu/)[17] for this research work. The dataset consists of 255 images, out of which 220 abnormal and 35 normal images have been employed for training, testing. Out of 255 images, 28 normal and 176 abnormal images are considered for training. The images will undergoes the preprocessing, K-Means segmentation for detection of tumor location and removal of noise. The details of the dataset has been presented in Table-1.

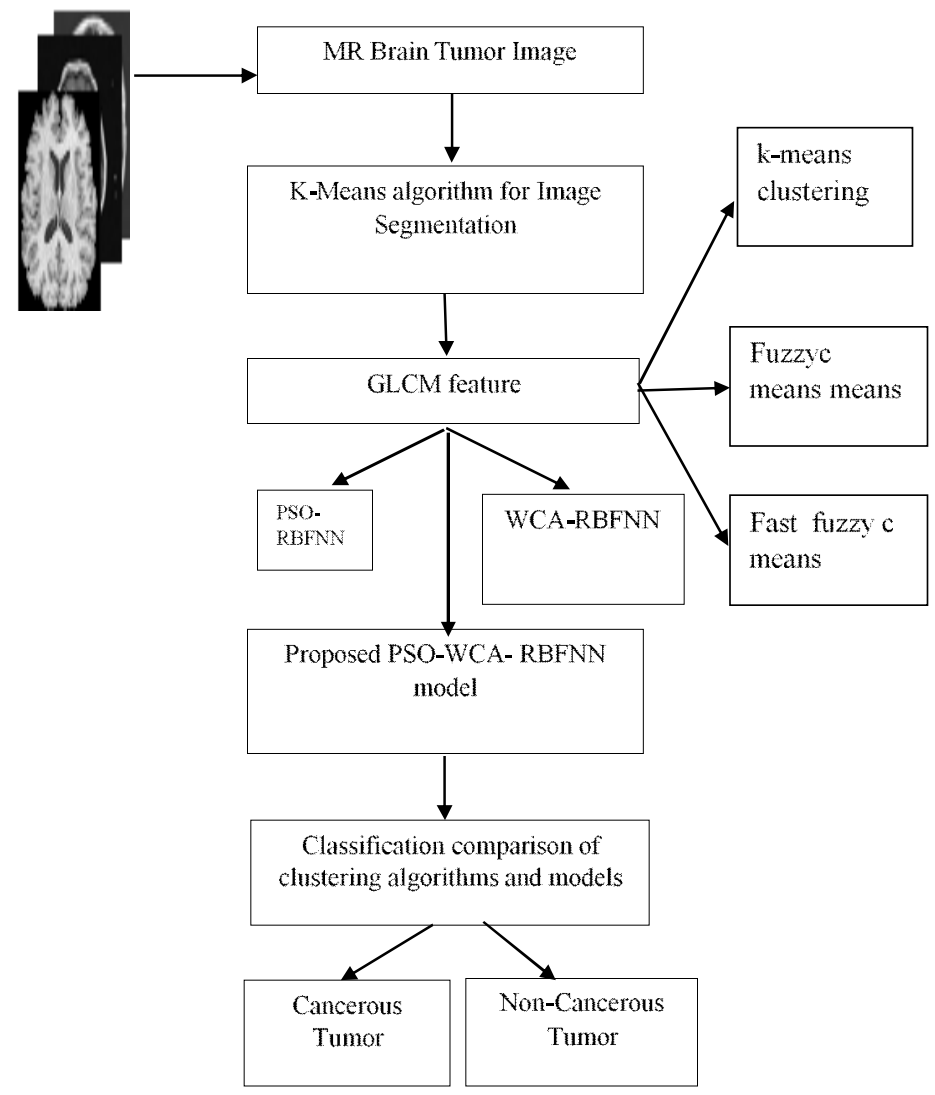

Figure1: Research Flow Diagram

Table -1: Dataset details

\begin{tabular}{lc}
\hline \multicolumn{2}{c}{ Dataset-255 } \\
\hline No. of images Normal & 35 \\
No. of images Abnormal & 220 \\
Training image Normal & 28 \\
Training image Abnormal & 176 \\
Images for testing Normal & 7 \\
Images for testing Abnormal & 44 \\
\hline
\end{tabular}

\subsection{Feature Extraction}

The features extracted by using the GLCM technique and the 
normalized features for 10 randomly chosen brain tumor MRI images are presented in Table-1. It is found that the features Variance versus kurtosis, skewness and energy are providing distinctive values for purpose of classification. A total of $255 \times 7=1785$ features are considered as input to the proposed Hybrid PSO-WCA-RBFNN classification model. Also the features are considered as input to the FCM, KNN [17] and Fast FCM algorithm for clustering classification of benign and malignant tumors.

\section{Table -1 Normalized Feature Extraction}

\begin{tabular}{|l|l|l|l|l|l|l|l|}
\hline Img. & $\begin{array}{l}\text { Std. } \\
\text { Dev }\end{array}$ & IDM & Entropy & Variance & Skewness & Kurtosis & Energy \\
\hline & 0.181 & 0.06 & 0.3127 & 0.0059 & 0.565 & 0.3232 & 0.4347 \\
\hline Im1 & 0.181 & 0.057 & 0.3271 & 0.0059 & 0.5657 & 0.3232 & 0.4349 \\
\hline Im2 & 0.14 & 0.029 & 0.1914 & 0.0031 & 0.4408 & 0.302 & 0.2134 \\
\hline Im3 & 0.14 & 0.037 & 0.2266 & 0.0034 & 0.7544 & 0.5253 & 0.2658 \\
\hline Im4 & 0.154 & 0.4151 & 0.0031 & 0.4915 & 0.3403 & 0.4132 \\
\hline Im5 & 0.162 & 0.057 & 0.3151 & 0.149 & 0.1052 & 0.1293 \\
\hline Im6 & 0.112 & 0.018 & 0.1129 & 0.0021 & 0.2149 & 0.1296 \\
\hline Im7 & 0.112 & 0.018 & 0.1229 & 0.0031 & 0.2148 & 0.1058 & 0.156 \\
\hline Im8 & 0.061 & 0.004 & 0.0397 & 0.0002 & 0.4703 & 0.1556 & 0.0309 \\
\hline Im9 & 0.147 & 0.032 & 0.2052 & 0.0031 & 0.9019 & 0.8422 & 0.2335 \\
\hline Im10 & 0.043 & 0.002 & 0.0207 & 0.0001 & 0.4284 & 0.13517 & 0.0144 \\
\hline & & & & & & & \\
\hline
\end{tabular}

\subsection{Fuzzy C Means Algorithm}

In Fuzzy C means clustering [18,27], the "cluster center $\mathrm{Ci}$ "and the "membership matrix U" are considered for distinct clusters. The objective function and center is presented as follows:

$J_{m}=\sum_{i=1}^{N} \sum_{j=1}^{C} u_{i j}^{m}\left\|x_{i}-c_{j}\right\|^{2}$

Where fuzziness coefficient $\mathrm{m}=2, u_{i j}$ represents the "degree of membership" of $x_{i}$ in cluster $j, x_{i}$ is the $i_{t h}$ of $n$-dimensional data, and $c_{j}$ is center of the cluster.

$c_{j}=\frac{\sum_{i=1}^{N} u_{i j}^{m} \cdot x_{i}}{\sum_{i=1}^{N} u_{i j}^{m}}, u_{i j}=\sum_{k=1}^{C}\left[\frac{\left\|x_{i}-c_{j}\right\|}{\left\|x_{i}-c_{k}\right\|}\right]^{-2 / m-1}$

\subsection{Fast Fuzzy C Means Algorithm}

According to Fast FCM algorithm[19] Let $X=\left[x_{1}, x_{2} \ldots \ldots x_{n}\right]$ be a $n$ sample data set and assume that each sample $x_{k}$ is represented by a set of $p$ features and $U$ is the hard partition matrices whose general term is given by $u_{i k}=1$ if $x_{k} \in X_{i}$, and 0 otherwise. To get partition matrix, the HCM (Hard c Means) algorithm is chosen which minimizes the objective function

$j=\sum_{k=1}^{n} \sum_{i=1}^{L} u_{i k}^{m}\left\|x_{k}-C_{i}\right\|^{2}$
Where "L" presents the number of clusters and $C_{i}$ is the cluster center and " $\mathrm{m}$ " is the Fuzzifier exponent and $u_{i k} \in[0,1]$.Minimization of equation (1) is obtained by an optimization technique that successively updates the cluster centers $C_{i}$ and partition matrix $U$ by using the formula.

$$
C_{i}=\frac{\sum_{k=1}^{n} u_{i k}^{m} x_{k}}{\sum_{k=1}^{n} u_{i k}^{m}}
$$

$$
\text { And } u_{i k}=\frac{1}{\sum_{j=1}^{L} \frac{\left\|x_{k}-C_{i}\right\|}{\left(\left\|x_{k}-C_{j}\right\|\right)^{2 /(m-1)}}}
$$

\subsection{Proposed PSO-WCA based RBFNN algorithm}

Fig.2 shows the structure of the RBFNN [20,21]. In this model, it is noticed that in RBFNN [21] model the input nodes and hidden nodes are taken as equal. In the RBFNN model, the weight is trained iteratively and weights has been assigned to the computational hidden node. This reduces the overall nodes requirement and offers better estimate to the task of classification.

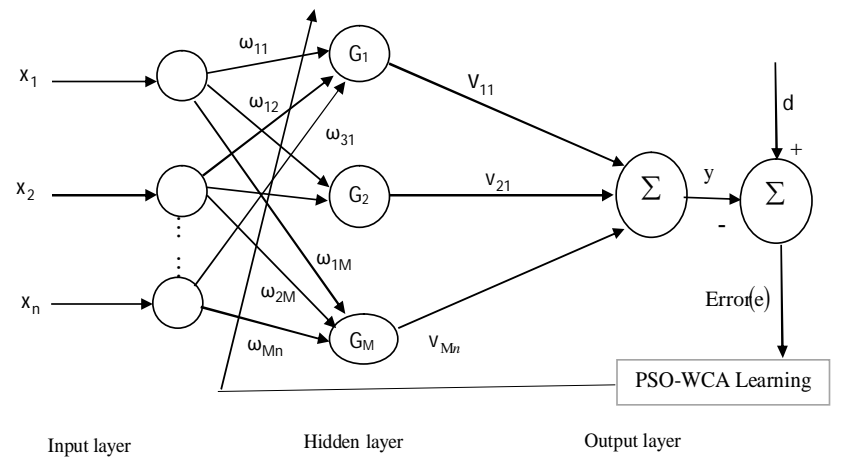

Fig: 2PSO-WCA Based Radial Basis Function Network

The activation function with Gaussian Kernel of the $\mathrm{M}^{\text {th }}$ node is given by

$G_{M}(x)=e^{\left(\frac{-\left\|x_{i}-C_{j}\right\|^{2}}{2 \sigma_{M}^{2}}\right)}$

Where $\sigma_{n}^{2}$ is controlling the smoothness and $\mathrm{C}_{\mathrm{j}}$ is center associated with hidden node and $\left\|x_{i}-c_{j}\right\|$ represents Euclidean distance.

The output layer is given by

$$
y=\sum_{n=1}^{N}\left(\mathrm{~V}_{11} x_{1}+\mathrm{V}_{12} x_{2}-----\mathrm{V}_{M n} x_{n}\right) \cdot e^{\left(\frac{-\|\left(x_{N}-C_{M} \|^{2}\right.}{2 \sigma_{M}{ }^{2}}\right)}
$$

The mean square error is the objective function and is given by

$\operatorname{MSE}(e)=\frac{1}{N} \sum_{n=1}^{N}\left(d_{n}-y_{n}\right)^{2}$

Where "d" is the desired vector. 
The weight optimization of RBFNN is proposed by utilizing the hybrid PSO and WCA algorithm. Considering PSO which uses a population of individuals in search space called particle and particles taken as features. The set of feature populations are called as swarm. The particles alters their mechanisms and fly in a search space.

The update equation for velocity is given by

$v_{i}(p+1)=\chi v_{i}(p)+R_{1}\left(\right.$ pbest $\left.(p)-x_{i}(p)\right)+R_{2}\left(\right.$ gbest $\left.(p)-x_{i}(p)\right)$

And the update equation for position is given by

$x_{i}(p+1)=x_{i}(t)+x_{i}(p+1)$

Where " $\chi$ " is the constriction factor and $R_{1}, R_{2}$ are random variables. Now considering the WCA[23] algorithm which clones the flow of streams and rivers into the sea in the form of a matrix of size " $\psi_{\text {Population }} \times d$ ", and " $d$ "represents the matrix dimension and is given by

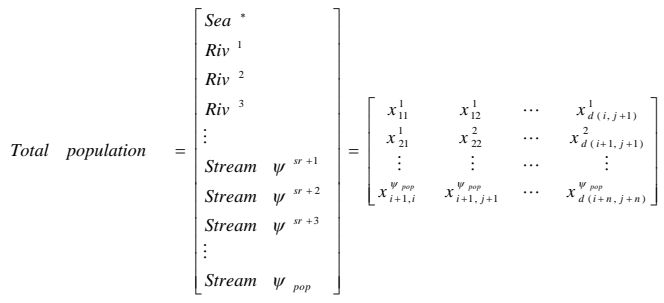

Where $\psi_{\text {Population }}$ is population size and $\psi_{s r}$ are selected values as the sea and (Riv)rivers

$\psi_{s r}=$ No. of rivers $+1($ sea $)$

$\psi_{\text {Stream }}=\psi_{\text {population }}-\psi_{s r}$

$\psi s_{n}=$ round $\left\{\left|\frac{f\left(R i v_{n}\right)}{\sum_{i=1}^{w_{N}} f\left(R i v_{i}\right)}\right| \times \psi_{\text {Stream }}\right\}, \quad n=1,2,3 \ldots \psi_{s t}$

Where, $\psi s_{n}$ is the "number of streams', and $f$ is the evaluation function in the algorithm. Now mapping with the position equation with $\vec{X}_{\text {Stream }}, \vec{X}_{\text {Sea }}, \vec{X}_{\text {River }}$, the best solutions are obtained by updating the WCA parameters.

The updated positions for "streams" and "rivers" have been evaluated as follows.

$$
\begin{aligned}
& x_{\text {Strean }(i, j)}(p+1)=x_{\text {Stream }(i, j)}(p)+\lambda \times\left(x_{\text {Sea }(i, j)}(p)-x_{\text {Strean }(i, j)}(p)\right) \\
& x_{\text {Strean }(i, j)}(p+1)=x_{\text {Strean }(i, j)}(p)+\lambda \times\left(x_{\text {River }(i, j)}(p)-x_{\text {Strean }(i, j)}(p)\right) \\
& x_{\text {River }(i, j)}(p+1)=x_{\text {River }(i, j)}(p)+\lambda \times\left(x_{\text {Sea }(i, j)}(p)-x_{\text {River }(i, j)}(p)\right)
\end{aligned}
$$

Where $\lambda$ is the controlling parameter and the velocity equation is updated by

$$
\begin{aligned}
& V_{\text {Srream }(i, j)}(p+1)=\zeta V_{\text {Srrean }(i, j)}(p)+R \times\left(\text { gbest }(p)-V_{\text {Sea }(i, j)}(p)-V_{\text {Srream }(i, j)}(p)\right) \\
& V_{\text {Srream }(i, j)}(p+1)=\zeta V_{\text {Srranari, } i, j}(p)+R \times\left(\text { gbest }(p)-V_{\text {Riverefi,j) }}(p)-V_{\text {Srream }(i, j)}(p)\right) \\
& V_{\text {River }(i, j)}(p+1)=\zeta V_{\text {River }(, j)}(p)+R \times\left(\text { gbest }(p)-V_{\text {Sea }(, j, j)}(p)-V_{\text {River }(i, j)}(p)\right)
\end{aligned}
$$

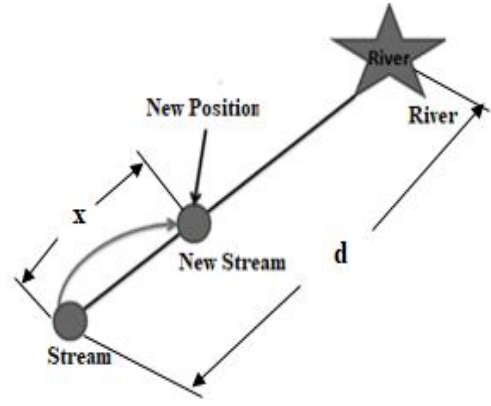

Fig. 3 Representation of streams flowing into a specific river

\subsection{Center updation using K- Means Algorithm}

The K-means[24] Clustering Algorithm starts by picking the number $\mathrm{K}$ of centres and randomly assigning the data points

$x_{i}$ to $S_{i}$ subsets containing $N_{j}$ data points that minimizes the cost function. It then uses a simple re-estimation procedure to end up with a partition of the data points into clusters containing $N$ data points that minimizes the sum squared clustering function. The clustering process terminates when no more data points switch from one cluster to another

$J_{m}=\sum_{j=1}^{K} \sum_{i \in S_{I}}\left\|x_{i}-c_{j}\right\|^{2}$

Where $c_{j}=\frac{1}{N_{j}} \sum_{i \in s_{j}} x_{i}$

This process choosing center and updation of center of RBFNN

Step1: Let " $X=\left\lfloor\left(x_{j 1}, x_{j 2}, \ldots, x_{j n}\right)\right\rfloor \quad j=1,2, \ldots, N$ " is the data set required to be clustered.

Step2: Initially take random centers and the data points as the input features.

Step 3: For every data point, the center finding the nearest mean to each data point, and reassigning the data points to the associated clusters $\mathrm{Cj}$, and then recomputing the cluster means as the corresponding center and updated by using K-Means algorithm.

Step 4: Repeat step-2 to step-3 for to get optimized center.

Step 5: The proposed PSO-WCA based RBFNN algorithm utilizes the optimized centers as to attain the essential clustering.

Step 7: The optimized centers are also sent as inputs to the fast fuzzy c means, Fuzzy c means, and KNN algorithm for the purpose of comparison with the proposed algorithm. 


\subsubsection{Pseudo code}

1. Initializing particles with random position and velocity vectors and WCA arameters $\lambda, \psi_{s r}, \psi_{\text {pop }}$

2. Initilize the weights of the RBFNN model

3. \%optimization loop

4.for $\mathrm{i}=1: \mathrm{k}$

for $\mathrm{j}=1: \mathrm{N}$

Update particles velocity and position equation (9) and (10) $\%$ update new parameter

$$
\begin{aligned}
& W_{\text {Stream }(i, j)}(p+1)=\zeta W_{\text {Stream }(i, j)}(p)+R \times\left(W_{\text {Sea }(i, j)}(p)-W_{\text {Stream }(i, j)}(p)\right) \\
& W_{\text {Stream }(i, j)}(p+1)=\zeta W_{\text {Stream }(i, j)}(p)+R \times\left(W_{\text {River }(i, j)}(p)-W_{\text {Stream }(i, j)}(p)\right) \\
& W_{\text {River }(i, j)}(p+1)=\zeta W_{\text {River }(i, j)}(p)+R \times\left(W_{\text {Sea }(i, j)}(p)-W_{\text {River }(i, j)}(p)\right) \\
& \text { and obtain fitness }
\end{aligned}
$$

5. end for the loop $\mathrm{i}$

6. end for the loop $\mathrm{j}$

7.Stop: update the weight till convergence to get fitness optimal solution, else repeat step-4 to step-7.

\section{RESULTS}

\subsection{Segmentation Results}
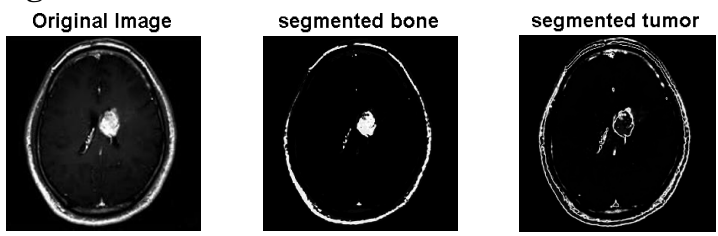

Fig:3Segmentation by using FCM
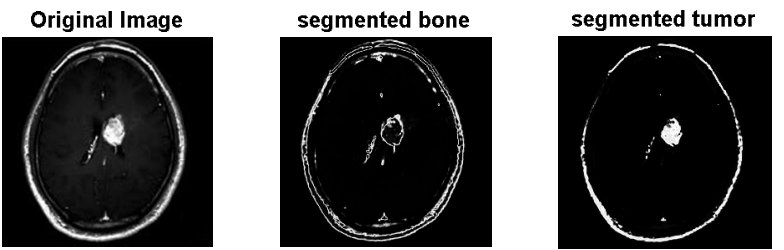

Fig:4Segmentation by using Fast FCM
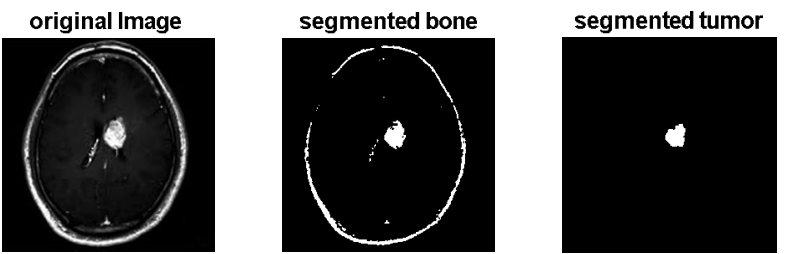

Fig.5Segmentation by using K-Means algorithm Table-2: Segmentation Accuracy of the model

\begin{tabular}{lcc} 
Algorithm & $\begin{array}{c}\text { Computatio } \\
\text { nal Time }\end{array}$ & $\begin{array}{c}\text { Accuracy } \\
\text { in \% }\end{array}$ \\
\cline { 2 - 3 } K-Means. & 11.1431 & $\mathbf{9 9 . 1 2}$ \\
Fuzzy C-Means & 14.7323 & 97.18 \\
Fast Fuzzy_C-Means & 19.1232 & 96.43 \\
\hline
\end{tabular}

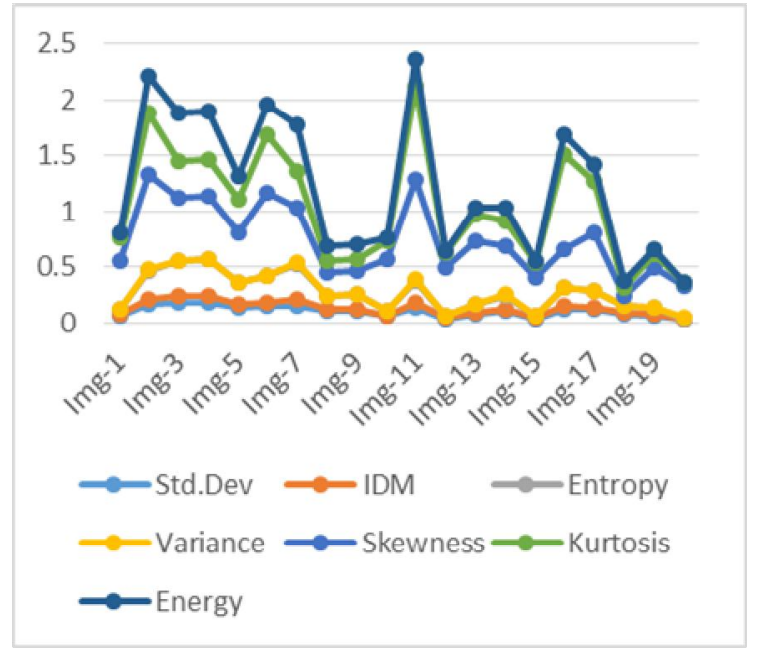

Fig.6Normalized Feature extraction plot of brain tumor

3.2 Classification clustering results

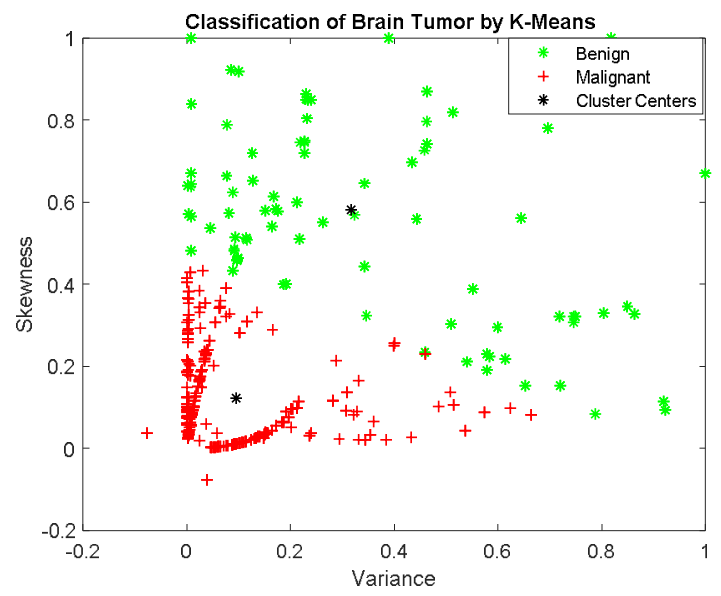

Fig.7 Classification of brain tumor using K- Means algorithm

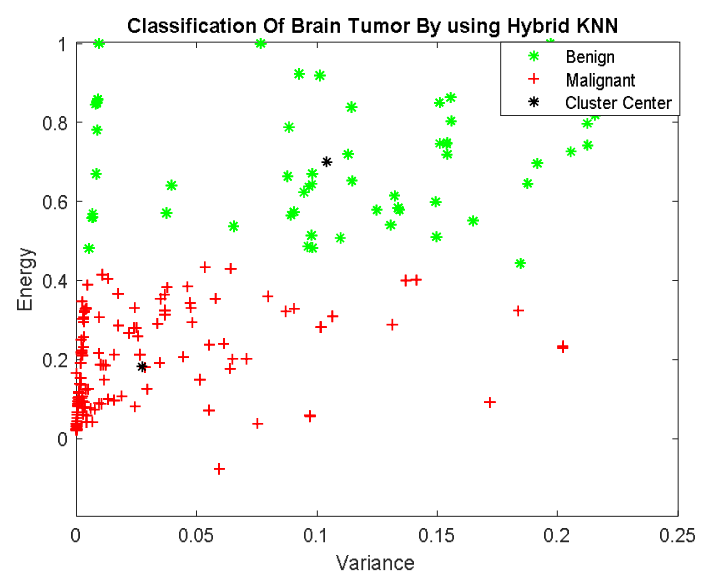

Fig.8 Classification of brain tumor using KNN algorithm 


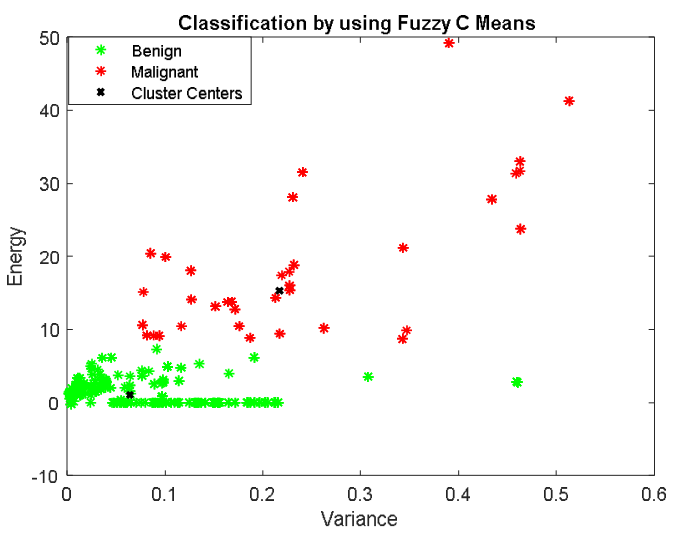

Fig.9 Classification of brain tumor using FCM algorithm

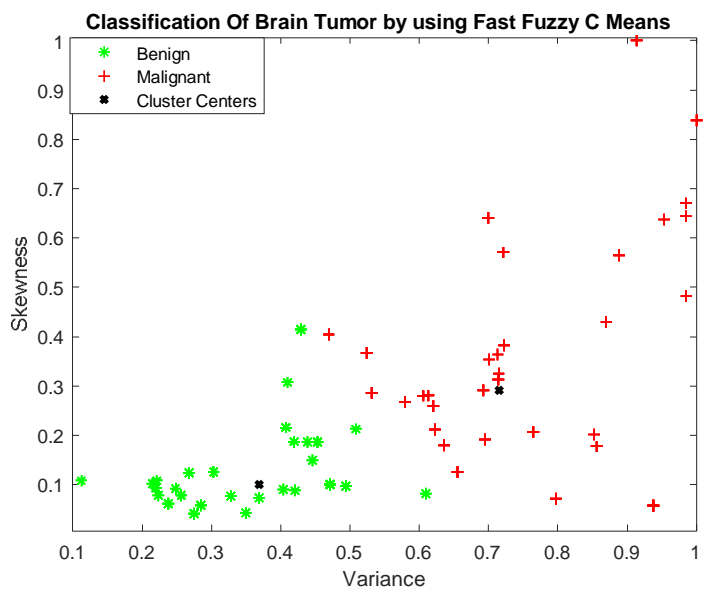

Fig.10 Classification of brain tumor using Hybrid PSO-WCA-RBFNN Model

\section{DISCUSSION}

Fig-3 to Fig-5 shows the segmentation process achieved by K-Means algorithm, Fast FCM and FCM. A total of 255 images are collected from Harvard medical school of architecture and taken for training and classification task. It found that the segmentation process clearly indicates the original brain tumor MRI, segmented brain and segmented tumor. It is found that the segmented tumor in case of K-Means algorithm shows better segmentation results and achieved an accuracy of $99.12 \%$. The segmentation results are presented in the Table-2. In the proposed work features such as variance, Entropy. IDM etc. have been reported in Table- 1 . The variance and entropy are found to be the most distinguished features which is presented in Fig-6. A total of $255 \times 7=1785$ features are considered for the clustering. Therefore, variance is taken as reference with entropy, kurtosis, skewness etc. for clustering. The features are submitted as input to the PSO-WCA based RBFNN algorithm for clustering classification. The simulation has been carried out with using MATLAB R2019a software, with 4GB RAM, CPU machine. Fig. 7 to Fig.10 shows the classification results by considering different feature combinations. The clustering accuracy have been obtained from the model and presented in the Table-3. Also the computational time has been calculated and presented. The mean square error plot in Fig.11 shows the robustness of the classification model. The proposed PSO-WCA-RBFNN model takes nearly 490 iterations, PSO-RBFNN takes 790 iterations, WCA-RBFNN takes 690 iterations and LMS-RBFNN takes 580 iterations for convergence. It is observed that the model RBFNN with PSO-WCA takes near about 12.217834 seconds for training and obtained $99.62 \%$ training accuracy when compared to other models. The classification accuracies of different models are presented in Table-4.

Table-3: Classification Accuracy of the model

\begin{tabular}{lccc}
\hline Model & No. of data & $\begin{array}{c}\text { Computational } \\
\text { Time }\end{array}$ & $\begin{array}{c}\text { Clustering } \\
\text { accuracy }\end{array}$ \\
\cline { 2 - 4 } KNN & 1785 & 32.124523 & 91.27 \\
K-MEANS & 1785 & 27.116431 & 96.29 \\
Fuzzy C & 1785 & 22.273292 & 97.12 \\
Fast Fuzzy C & 1785 & 19.123242 & 98.49 \\
Means & & & \\
\hline
\end{tabular}

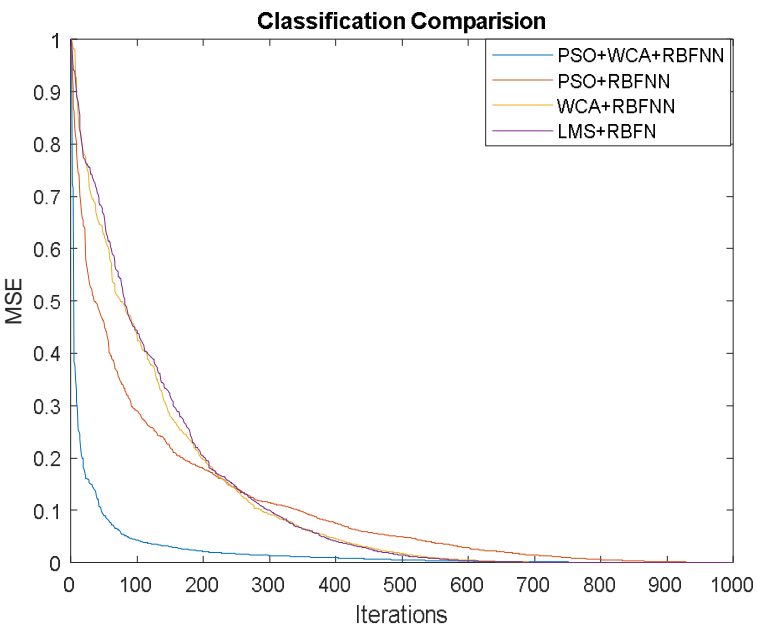

Fig.11 Mean Square Error comparison of models

\section{CONCLUSION}

The research work shows a better clustering results by considering the two popular types of tumors such as as benign and malignant for classification through clustering. Feature extraction has been accomplished by GLCM technique and image segmentation by utilizing FCM, Fast FCM and k-means algorithm. The proposed PSO based RBFN model has shown the potentiality of clustering of the tumor. The automatic detection and classification using the proposed RBFNN model with PSO-WCA training is the main contribution of the research work. The feature variance played a vital role in clustering classification in comparison to the other features. The feature variance have given adequate classification results with kurtosis with variance, variance with skewness, and variance with energy. The proposed 
RBFNN with PSO-WCA model has been assigned for the classification and the results were compared with the PSO-RBFNN,WCA-RBFNN and LMS-RBFNN approach. From the result it is found that the proposed model provides better classification result and the computations time obtained as less as compared to other mentioned methods.

\section{REFERENCES}

1. https://www.mayoclinic.org/diseases-conditions/brai n-tumor/symptoms-causes/syc-20350084,2020.

2. Mohd Fauzi Bin Othman, Noramalina Bt Abdullah, MRI Brain Classification using Support Vector Machine,Fourth International Conference on Modeling, Simulation and Applied Optimization IEEE, 2011

3. Mohd Fauzi Othman and Mohd Ariffanan Mohd Basri, Probabilistic Neural Network for Brain Tumor Classification, Second International Conference on Intelligent Systems, Modelling and Simulation. IEEE, ISMS.2011.32, 2011.

4. S. Damodharan and D. Raghavan, Combining tissue segmentation and neural network for brain tumor detection, International Arab Journal of Information Technology, Vol. 12, no. 1, pp. 42-52, 2015.

5. M. Alfonse and A.-B. M. Salem, An automatic classificati on of brain tumors through MRI using support vector machine, Egyptian Computer Science Journal, Vol. 40, pp. 11-21, 2016.

6. P. Kumar and B. Vijayakumar, Brain tumour $\mathbf{M r}$ image segmentation and classification using by PCA and RBF kernel based support vector machine, Middle-East Journal of Scientific Research, vol. 23, no. 9, pp. 2106-2116, 2015.

7. W. Cui, Y. Wang, Y. Fan, Y. Feng, and T. Lei, Localized FCM clustering with spatial information for medical image segmentation and bias field estimation, International Journal of Biomedical Imaging, vol. 2013, Article ID 930301,, 2013.

8. N. Sharma, A. Ray, S. Sharma, K. Shukla, S. Pradhan, and L. Aggarwal, Segmentation and classification of medical images using texture-primitive features: application of BAM-type artificial neural network, Journal of Medical Physics, vol. 33, no. 3, pp. 119-126, 2008.

9. E. A. Zanaty, Determination of gray matter (GM) and white matter (WM) volume in brain magnetic resonance images (MRI), International Journal of Computer Applications, vol. 45, pp. 16-22, 2012

10. G. Wang, J. Xu, Q. Dong, and Z. Pan, Active contour model coupling with higher order diffusion for medical image segmentation, International Journal of Biomedical Imaging, Vol. 2014, Article ID 237648, 8 pages, 2014.

11. T. Torheim, E. Malinen, K. Kvaal, Classification of dynamic contrast enhanced MR images of cervical cancers using texture analysis and support vector machines, IEEE Transactions on Medical Imaging, vol. 33, no. 8, pp. 1648-1656, 2014.

12. S. N. Deepa and B. Arunadevi, Extreme learning machine for classification of brain tumor in 3DMR images, Informatologia, vol. 46, no. 2, pp. 111-121, 2013.

13. A. Chaddad, Automated feature extraction in brain tumor by magnetic resonance imaging using gaussian mixture models, International Journal of Biomedical Imaging, Vol. 2015, Article ID 868031 .

14. N. B. Bahadure, A. K. Ray, H. P. Thethi, Image Analysis for MRI Based Brain Tumor Detection and Feature Extraction Using Biologically Inspired BWT and SVM, Hindawi International Journal of Biomedical Imaging, Volume 2017, Article ID 9749108, https://doi.org/ 10.1155/ 2017/ 9749108.

15. P. Mohanaiah, P. Sathyanarayana, L. GuruKumar, Image texture feature extraction using GLCM approach, International Journal of Scientific and Research Publications, Volume 3, Issue 5, May 2013

16. N. Hema Rajini, R.Bhavani, Classification of MRI Brain Images using k- Nearest Neighbor and Artificial Neural Network, International Conference on Recent Trends in Information Technology. IEEE, 2011.

17. Maryam Kuhkan, A Method to Improve the Accuracy of K-Nearest Neighbor Algorithm ,International Journal of Computer Engineering and Information Technology Vol. 8, NO. 6, June 2016, 90-95

18. Satyasis Mishra, Premananda Sahu \& Manas Ranjan Senapati, MASCA- PSO based LLRBFNN Model and Improved fast and robust FCM algorithm for Detection and Classification of 
Brain Tumor from MR Image, Evolutionary Intelligence, 12, 647-663 (2019) Springer https:// doi.org/ 10.1007/s12065-019-00266-x,July,2019

19. D.Mújica-Vargas, J.M.V. Kinani, \& J.d. Rubio, Color-Based Image Segmentation by Means of a Robust Intuitionistic Fuzzy C-means Algorithm. Int. J. Fuzzy Syst. 22, 901-916 (2020). https://doi.org/10.1007/s40815-020-00824-x

20. P. K. Nayak, S. Mishra, P. K. Dash, Ranjeeta Bisoi, Comparison of modified teaching-learning-based optimization and extreme learning machine for classification of multiple power signal disturbances, Neural Computing \& Application, Springer,2016. DOI 10.1007/s00521 -015-2010-0

21. T. McConaghy, H. Lung, E. Bosse, V. Vardan, Classification Of Audio Radar Signals Using Radial Basis Function Neural Network, IEEE Transactions on Inst .And Measurement, Vol. 52, No.6, pp.1771-17779, Dec. 2003.

22. N. Najkar, F. Razzazi, H. Sameti., A Novel Approach To HMM-based Speech Recognition Systems Using Particle Swarm Optimization, Elsevier Science, Mathematical And Computer Modeling, Vol.52, No.11, pp.2157-2165, Dec.2010

23. A Sadollah., H Eskandar., JH Kim, WCA for solving constrained multi-objective optimization problems., Appl. Soft Comput. Vol.27, pp.279-289 ,2015.

24. H.Zare,S.Emadi, Determination of Customer Satisfaction using Improved K-means algorithm. Soft Comput (2020). https://doi.org /10.1007/s00500-020-04988-4.

25. Sreelekha Panda, Satyasis Mishra, Manas Ranjan Senapati ,Detection and Classification Methods for EEG Epileptic Seizures, International Journal of Advanced Trends in Computer Science and Engineering,(2019), , Volume 8, No.6, November Dec. 2019, 2925- 2934., https://doi.org/ 10.30534/ ijatcse/2019/40862019

26. S.Sankar Ganesh, S.V.Sudha, S.Ayyasamy, G.Pandiya Rajan, S.Chidambaram, Optimal Feature Subset Selection and MSVM Classification Based CBIR for Medical Images, International Journal of Advanced Trends in Computer Science and Engineering Volume 9, No.4, July - August 2020, https:// doi.org /10.30534 /ijatcse /2020/81942020.
27. D. Sherlin and D. Murugan, Brain Tumor segmentation using modified Fuzzy metric based Approach with Adaptive Technique, International Journal of Advanced Trends in Computer Science and Engineering Volume 8, No.6, November December 2019, https://doi.org /10.30534 /ijatcse/ 2019/08862019.

28. Jide E. T. Akinsola, Moruf A. Adeagbo and Ayomikun A. Awoseyi, Breast Cancer Predictive Analytics Using Supervised Machine Learning Techniques, International Journal of Advanced Trends in Computer Science and Engineering, Volume 8, No.6, November - December 2019, https://doi.org/10.30534/ijatcse/2019/29862019 